\title{
Nonlinear Stability of Solitary Waves of a Generalized Kadomtsev-Petviashvili Equation
}

\author{
Yue Liu', Xiao-Ping Wang ${ }^{2}$ \\ ${ }^{1}$ Department of Mathematics, University of Texas at Austin Austin, TX 78712, USA \\ 2 Department of Mathematics, The Hong Kong University of Science and Technology, \\ Clear Water Bay, Kowloon, Hong Kong
}

Received: 12 December 1995/Accepted: 6 June 1996

Abstract: We prove that the set of solitary wave solutions of a generalized Kadomtsev-Petviashvili equation in two dimensions,

$$
\left(u_{t}+\left(u^{m+1}\right)_{x}+u_{x x x}\right)_{x}=u_{y y}
$$

is stable for $0<m<4 / 3$.

\section{Introduction}

The generalized Kadomtsev-Petviashvili (GKP) equation

$$
\left(u_{t}+\left(u^{m+1}\right)_{x}+u_{x x x}\right)_{x}=\sigma^{2} u_{y y}
$$

is a two dimensional analog of the generalized Korteweg-de Vries (GKdV) equation. When $m=1$ and $\sigma^{2}=1,(1)$ is known as the KPI equation while $m=1$ and $\sigma^{2}=-1$ corresponds to the KPII equation. Both are universal models for the propagation of weakly nonlinear dispersive long waves which are essentially one directional, with weak transverse effects [6]. It also describes the evolution of sound waves in antiferromagnetics [9]. It is well known that both KPI and KPII can be solved by the Inverse Scattering Transformation (IST) [1, 2].

Many local existence results for KP and GKP have recently appeared for both infinite space and periodic boundary conditions (see $[19,20,13])$. There are also some global results [22]. It is shown in $[9,20]$ by a virial method that GKP-I

$$
\left(u_{t}+\left(u^{m+1}\right)_{x}+u_{x x x}\right)_{x}=u_{y y}
$$

has blow-up solutions for $m \geqq 4$ while arguments in [14] indicate that blow up should occur for much lower $m$, namely $m>\frac{4}{3}$.

An important question is the stability and instability of solitary waves for GKP, that is, solutions of form $u(x, y, t)=\varphi(x-c t, y)$. Existence of solitary waves is shown in [14] for $1<m<4$ and also in [21] by a different method. For GKP-I, instability is shown in [14] for $\frac{4}{3}<m<4$ using a method of Shatah and Strauss [3] and a completed proof is provided by de Bouard and Saut [24]. In this paper, we shall prove that the solitary waves are nonlinearly stable for $0<m<\frac{4}{3}$. After this 
paper was completed, we learned that de Bouard and Saut [24] have a similar result by a different method.

The paper is organized as follows. In Sect. 2, we give the detailed proof of the existence of solitary waves for GKP-1 with $0<m<4$. The solutions are obtained by using the variational method and the techniques developed by Lieb [18] to solve a constrained minimization problem. In Sect. 3, we prove the set of solitary waves of KP-I is nonlinearly stable for $0<m<\frac{4}{3}$. The proof is based on the idea of Shatah [4] and Levandosky [5]. We use the variational properties of the minimizer and a convexity lemma of Shatah to estabilish the key inequality for stability theorem.

We shall use the following notations. $|\cdot|_{p}$ (resp. $\|\cdot\|_{s}$ ) will stand for the norm in the Lebesque space $L^{p}\left(\mathbf{R}^{2}\right)$ (resp. the Sobolev space $H^{s}\left(\mathbf{R}^{2}\right)$ ) .

Because of the structure of Eq. (1), we introduce the following function space:

$$
V(\Omega)=\left\{u \mid u \in L^{2}(\Omega), u_{x} \in L^{2}(\Omega), D_{x}^{-1} u_{y} \in L^{2}(\Omega)\right\}
$$

equipped with a norm

$$
|u|_{V}=\left(\int_{\Omega}\left(u^{2}+|\tilde{\nabla} u|^{2}\right) d x d y\right)^{\frac{1}{2}}
$$

and an inner product

$$
\langle u, v\rangle=\int_{\Omega}(u v+\tilde{\nabla} u \cdot \tilde{\nabla} v) d x d y
$$

Here $\Omega$ may be $\mathbf{R}^{2}$ or a box $[a, b] \times[c, d]$ in $\mathbf{R}^{2}$, and $\tilde{\nabla} u=\left(u_{x}, D_{x}^{-1} u_{y}\right)^{T}$, $D_{x}^{-1}=\int_{-\infty}^{x}\left(\right.$ or $\left.\int_{-a}^{x}\right)$.

We thank the referee for the vaulable comments.

\section{Existence of Solitary Waves}

In this section, we give a more detailed proof of the existence of localized solitary waves for GKP-I given in [14]. By solitary waves, we mean traveling wave solutions of the form $u(x, y, t)=\varphi_{\omega}(x-\omega t, y)$. Substituting in (1), $\varphi_{\omega}$ satisfies the following equation:

$$
\omega \varphi+D_{x}^{-2} \varphi_{y y}-\varphi_{x x}=\varphi^{m+1} .
$$

For $m=1$, this equation has an explicit solution (lump soliton [1])

$$
v(x, y)=8 \frac{\omega-x^{2} / 3+y^{2} /(3 \omega)}{\left(\omega+x^{2} / 3+y^{2} /(3 \omega)\right)^{2}}
$$

We shall prove the existence of decaying solutions for $0<m<4$ in space $V=V\left(\mathbf{R}^{2}\right)$ by a variational method. We first introduce the following functionals on $V\left(\mathbf{R}^{2}\right)$,

$$
\begin{gathered}
Q(u)=\frac{1}{2} \int_{\mathbf{R}^{2}} u^{2} d x d y, \\
E(u)=\int_{\mathbf{R}^{2}}\left(\frac{1}{2}\left(\left(D_{x}^{-1} u_{y}\right)^{2}+u_{x}^{2}\right)-\frac{1}{(m+2)} u^{m+2}\right) d x d y
\end{gathered}
$$




$$
\begin{gathered}
K(u)=\int_{\mathbf{R}^{2}} u^{m+2} d x d y, \\
I_{\omega}(u)=\int_{\mathbf{R}^{2}}\left(\omega u^{2}+|\tilde{\nabla} u|^{2}\right) d x d y .
\end{gathered}
$$

Remark 1. Here and in the following, we always assume that $m=m_{1} / m_{2}$, where $m_{1}$ is any even integer and $m_{2}$ any odd integer. This guarantees that $K(u)$ is nonnegative.

Let's also define the following minimization problem:

$$
M(\omega)=\inf _{u \in V\left(\mathbf{R}^{2}\right)} \frac{I_{\omega}(u)}{(K(u))^{\frac{2}{m+2}}} .
$$

It is easy to see that

$$
\frac{I_{\omega}(\lambda u)}{(K(\lambda u))^{\frac{2}{m+2}}}=\frac{I_{\omega}(u)}{(K(u))^{\frac{2}{m+2}}} \text { for } \lambda \neq 0
$$

Similarly, we have

$$
M(\omega)=\inf _{u \in V}\left\{I_{\omega}(u) \mid K(u)=1\right\}
$$

By change of variable $u(x, y)=w^{\frac{1}{m}} V(\sqrt{w} x, w y)$, one easily obtains that

$$
M(w)=w^{\frac{4-m}{2(m+2)}} M(1) \quad w>0 .
$$

Note that Eq. (4) is the Euler-Lagrange equation of the functional

$$
L_{\omega}(u)=\int\left(\frac{\omega}{2} u^{2}+\frac{1}{2}|\tilde{\nabla} u|^{2}-\frac{1}{(m+1)(m+2)} u^{m+2}\right) d x d y .
$$

Therefore, if there is a function $\varphi \in V\left(\mathbf{R}^{2}\right)$, such that

$$
I_{\omega}(\varphi)=M(\omega) \text { with } K(\varphi)=1,
$$

then $\varphi$ is a solution of

$$
\omega \varphi-\varphi_{x x}+D_{x}^{-2} \varphi_{y y}=\lambda \varphi^{m+1}
$$

where $\lambda$ is the Lagrange multiplier. Hence $\psi=\lambda^{\frac{1}{m}} \varphi$ is the solution of (4). We call such a solution a ground state.

Theorem 1 (Existence of solitary waves). Let $0<m<4, \omega>0$ and $m=m_{1} / m_{2}$, where $m_{1}$ it is any even integer and $m_{2}$ any odd integer. Then there exists $a$ minimizer $\varphi_{w} \in N$ such that

$$
I_{\omega}\left(\varphi_{\omega}\right)=\inf _{u \in N} I_{\omega}(u)=M(\omega)
$$

where $N=\left\{u \mid u \in V\left(\mathbf{R}^{2}\right), K(u)=1\right\}$ and $K(u)$ given by $(8)$.

To prove this theorem, we use the techniques used in [15, 18, 23 and 17]. The following lemmas are needed for the proof of Theorem 1 . We shall prove the lemmas later. 
Lemma 1. Let $V(\Omega)$ be the space defined in (3) and $\Omega$ may be $\mathbf{R}^{2}$ or a box in $\mathbf{R}^{2}$. Then for any $2<n<6$, there exists, a constant $C$, such that for any $u \in V(\Omega)$,

$$
\left(\int_{\Omega}|u|^{n}\right)^{\frac{1}{n}} \leqq C\left(\int_{\Omega}|\tilde{\nabla} u|^{2}+u^{2}\right)^{\frac{1}{2}} .
$$

Lemma 2. Let $u \in V(B)$, where $B \subset \mathbf{R}^{2}$ is an arbitary box. Then $\forall \varepsilon>0$, there exist integers $N_{\varepsilon}, M_{\varepsilon}$, s.t.

$$
\int_{B} u^{2} \leqq \sum_{n_{1}=1}^{M_{\varepsilon}} \sum_{n_{2}=1}^{N_{\varepsilon}}\left(\int_{B} u w_{n_{1}, n_{2}}\right)+\varepsilon \int_{B}|\tilde{\nabla} u|^{2},
$$

where $w_{n_{1}, n_{2}}$ are orthonomal basis functions in $V(B)$.

Lemma 3. Let $\left\{u^{j}\right\}$ denote a minimizing sequence of $I_{1}=I_{\omega=1}$. That is, $\lim _{j \rightarrow \infty} I_{1}\left(u^{j}\right)=\inf _{u \in N} I_{1}(u)$. Then there exist $\varepsilon, \delta>0$ such that for all $j$,

$$
\mu\left(\left[\left|u^{j}\right|>\varepsilon\right]\right) \geqq \delta,
$$

where $\mu(\cdot)$ denotes the Lebesgue measure.

Lemma 4. Let $u$ be a function such that $|u|_{V} \leqq C$ and $\mu([|u|>\varepsilon]) \geqq \delta>0$. Then there exists a shift $T_{s, t} u(x, y)=u(x+s, y+t)$, such that for some constant $\alpha=\alpha(C, \delta, \varepsilon)>0$,

$$
\mu\left(B \cap\left[\left|T_{s, t} u\right|>\varepsilon / 2\right]\right)>\alpha,
$$

where $B$ is the unit box (i.e. box centered at origin and has length $1 \times 1$ ) in $\mathbf{R}^{2}$.

Lemma 2 is used in [11]. Lemmas 3 and 4 are similar to the results in [15 and 18].

Proof of Theorem 1. By (10), it suffices to show that there exists a minimizer $u_{0} \in N$ such that $I_{1}\left(u_{0}\right)=M(1)$. We denote $I(u)=I_{1}(u)$ and $I^{0}=\inf _{u \in N} I(u)$. Let $\left\{u_{j}\right\}$ be a minimizing sequence, i.e $I\left(u_{j}\right) \rightarrow I^{0}$ with $I\left(u_{j}\right) \leqq C,\left|u_{j}\right|_{m+2}=1$. We then have $u_{j} \rightarrow u_{0}$ weakly in $V, u_{j} \rightarrow u_{0}$ weakly in $L^{m+2}$. It follows from Lemma 2 , $u_{j} \rightarrow u_{0}$ strongly in $L^{2}$ on any bounded domain. And $u_{j} \rightarrow u_{0}$ a.e. in $\mathbf{R}^{2}$.

We next show $u_{j} \rightarrow u_{0}$ strongly in $L^{m+2}$. To do that it suffices to prove $\left|u_{0}\right|_{L^{m+2}}=1$; i.e. that $u_{0} \in N$. Since $u_{j} \rightarrow u_{0}$ weakly in $L^{m+2}$, this implies $\left|u_{0}\right|_{L^{m+2}} \leqq 1$. Next, we want to show that $\left|u_{0}\right|_{L^{m+2}} \neq 0$, i.e. $u_{0} \neq 0$. From Lemmas 3 and 4 , there exists $\alpha$, such that

$$
\mu\left(B \cap\left[\left|T_{s_{j}, t_{j}} u_{j}\right|>\varepsilon\right]\right)>\alpha .
$$

Let $T_{s_{j}, t_{j}} u_{j}$ be the new sequence denoted also by $u_{j}$, we have

$$
\mu\left(B \cap\left[\left|u_{j}\right|>\varepsilon\right]\right)>\alpha .
$$

Since $u_{j} \rightarrow u_{0}$ a.e., it follows that $\mu\left(B \cap\left[\left|u_{0}\right|>\frac{\varepsilon}{2}\right]\right)>\alpha$, therefore $u_{0} \neq 0$ and $\left\|u_{0}\right\|_{L^{m+2}} \neq 0$.

Next, we show that if $0<\lambda=\int u_{0}^{m+2}<1$, we have a contradiction. Denote $v_{j}=u_{j}-u_{0}$, so that $v_{j} \rightarrow 0$ weakly in $L^{m+2}$. Observe that due to a theorem of Brezis and Lieb (a refined Fatou lemma [16])

Note that

$$
\int\left|v_{j}\right|^{m+2} \rightarrow 1-\int u_{0}^{m+2}=1-\lambda .
$$

$I\left(u_{j}\right)=I\left(v_{j}+u_{0}\right)=I\left(v_{j}\right)+I\left(u_{0}\right)+2 \int v_{j x} u_{0_{x}}+2 \int\left(D_{x}^{-1} v_{j y}\right)\left(D_{x}^{-1} u_{0 y}\right)+2 \int v_{j} u_{0}$.

The last three terms converge to zero by weak convergence of $v_{j} \rightarrow 0$ in $V\left(\mathbf{R}^{2}\right)$. 
Hence

$$
I^{0}=\lim _{j \rightarrow \infty} I\left(v_{j}\right)+I\left(u_{0}\right) .
$$

Let $\tilde{u}_{0}=\frac{u_{0}}{\lambda \frac{1}{m+2}}$, then we have $\int\left|\tilde{u}_{0}\right|^{m+2}=1$. By homogeneity,

$$
I\left(u_{0}\right)=\lambda^{\frac{2}{m+2}} I\left(\frac{u_{0}}{\lambda^{\frac{1}{m+2}}}\right)=\lambda^{\frac{2}{m+2}} I\left(\tilde{u}_{0}\right) \geqq \lambda^{\frac{2}{m+2}} I^{0} .
$$

Let $\tilde{v}_{j}=\frac{v_{j}}{(1-\lambda)^{\frac{1}{m+2}}}$. From (13), we have $\int\left|\tilde{v}_{j}\right|^{m+2}=\frac{\int\left|v_{j}\right|^{m+2}}{1-\lambda} \rightarrow 1$. Similarly,

$$
\begin{gathered}
I\left(v_{j}\right)=(1-\lambda)^{\frac{2}{m+2}} I\left(\frac{v_{j}}{(1-\lambda)^{\frac{1}{m+2}}}\right)=(1-\lambda)^{\frac{2}{m+2}} I\left(\tilde{v}_{j}\right), \\
\lim _{j \rightarrow \infty} I\left(v_{j}\right)=(1-\lambda)^{\frac{2}{m+2}} \lim _{j \rightarrow \infty} I\left(\tilde{v}_{j}\right) \geqq(1-\lambda)^{\frac{2}{m+2}} I^{0} .
\end{gathered}
$$

Therefore

$$
I^{0}-I\left(u_{0}\right) \geqq(1-\lambda)^{\frac{2}{m+2}} I^{0} .
$$

Equations (15) and (16) give

$$
I^{0} \geqq\left(\lambda^{\frac{2}{m+2}}+(1-\lambda)^{\frac{2}{m+2}}\right) I^{0}>I^{0},
$$

which is a contradiction. Therefore

$$
\lambda=\int\left|u_{0}\right|^{m+2}=1
$$

i.e. $u_{j} \rightarrow u_{0}$ strongly in $L^{m+2}$.

Moreover, because $u_{j} \rightarrow u_{0}$ weakly in $V, I\left(u_{0}\right) \leqq$ inf $I\left(u_{j}\right)$ and $u_{0}$ minimizes $I$ in $N$. Hence $I\left(u_{j}\right) \rightarrow I\left(u_{0}\right)$. Therefore $u_{j} \rightarrow u_{0}$ strongly in $V\left(\mathbf{R}^{2}\right)$, and this establishes Theorem 1.

We now prove the lemmas.

Proof of Lemma 1. We shall prove (12) for $\Omega=\mathbf{R}^{2}$ only. The case with $\Omega$ being a box can be proved similarly. Consider the Fourier transform representations of $u, u_{x}$ and $D_{x}^{-1} u_{y}$,

$$
\begin{gathered}
u=\int \hat{u}(p, q) e^{i p x+i q y} d p d q, \quad u_{x}=\int \widehat{u_{x}}(p, q) e^{i p x+i q y} d p d q, \\
D_{x}^{-1} u_{y}=\int \widehat{D_{x}^{-1} u_{y}}(p, q) e^{i p x+i q y} d p d q
\end{gathered}
$$

Then, we have

$$
\int|u|^{2} d x d y=\int|\hat{u}|^{2} d p d q, \quad \int|\tilde{\nabla} u|^{2} d x d y=\int|\widehat{\tilde{\nabla} u}|^{2} d p d q .
$$

For some $0 \leqq \alpha \leqq 1$ and $p, q \neq 0$, we have

$$
\begin{aligned}
\hat{u}(p, q) & =\int e^{-i p x-i q y} u(x, y) d x d y \\
& =\alpha \int e^{-i p x-i q y} u(x, y) d x d y+(1-\alpha) \int e^{-i p x-i q y} u(x, y) d x d y \\
& =\frac{\alpha}{-i p} \int e^{-i p x-i q y} u_{x}(x, y) d x d y+\frac{(1-\alpha) p}{q} \int e^{-i p x-i q y} D_{x}^{-1} u_{y} d x d y .
\end{aligned}
$$


It follows that

$$
\begin{aligned}
|\hat{u}(p, q)| & \leqq \frac{\alpha}{|p|}\left|\widehat{u_{x}}\right|+\frac{(1-\alpha)|p|}{|q|}\left|\widehat{D_{x}^{-1}} u_{y}\right| \\
& \leqq \sqrt{\left(\frac{\alpha}{p}\right)^{2}+\left(\frac{(1-\alpha) p}{q}\right)^{2}} \cdot \sqrt{\left|\widehat{u_{x}}\right|^{2}+\left|\widehat{D_{x}^{-1} u_{y}}\right|^{2}} .
\end{aligned}
$$

Let $\alpha=\frac{p^{2}}{|q|+p^{2}}$. Then

$$
\begin{aligned}
|\hat{u}| & \leqq \frac{\sqrt{2}|p|}{|q|+p^{2}} \sqrt{\left|\widehat{u_{x}}\right|^{2}+\left|\widehat{D_{x}^{-1} u_{y}}\right|^{2}} \\
|\hat{u}|^{m} & \leqq\left(\frac{\sqrt{2}|p|}{|q|+p^{2}}\right)^{m}\left(\left|\widehat{u_{x}}\right|^{2}+\left|D_{x}^{-1} u_{y}\right|^{2}\right)^{\frac{m}{2}} .
\end{aligned}
$$

From Hölder's inequality, we have

$$
\begin{aligned}
& \int_{p^{2}+q^{2} \geqq 1}|\hat{u}|^{m} d p d q \leqq \\
& \int_{p^{2}+q^{2} \geqq 1}\left(\frac{|p|}{|q|+p^{2}}\right)^{m}\left\{\left|\widehat{u_{x}}\right|^{2}+\mid \widehat{\left.\left.D_{x}^{-1} u_{y}\right|^{2}\right\}^{\frac{m}{2}} d p d q}\right. \\
& \leqq\left(\int_{p^{2}+q^{2} \geqq 1}\left(\frac{|p|}{|q|+p^{2}}\right)^{\frac{2 m}{2-m}} d p d q\right)^{\frac{2-m}{2}} \\
& \times\left\{\int_{p^{2}+q^{2} \geqq 1}\left|\widehat{u_{x}}\right|^{2}+\left|\widehat{D_{x}^{-1} u_{y}}\right|^{2} d p d q\right\}^{\frac{m}{2}} .
\end{aligned}
$$

It is easy to see that $\int_{p^{2}+q^{2} \geqq 1}\left(\frac{|p|}{|q|+p^{2}}\right)^{\frac{2 m}{2-m}} d p d q$ is convergent if $\frac{6}{5}<m<2$. Hence

$$
\begin{aligned}
\int_{p^{2}+q^{2} \geqq 1}|\hat{u}|^{m} d p d q & \leqq C_{1}\left\{\int_{p^{2}+q^{2} \geqq 1}\left|\widehat{u_{x}}\right|^{2}+\left|\widehat{D_{x}^{-1} u_{y}}\right|^{2} d p d q\right\}^{\frac{m}{2}} \\
& \leqq C_{1}\left\{\int_{\mathbf{R}^{2}}\left|\widehat{u_{x}}\right|^{2}+\left|\widehat{D_{x}^{-1} u_{y}}\right|^{2} d p d q\right\}^{\frac{m}{2}},
\end{aligned}
$$

where $C_{1}=\int_{p^{2}+q^{2} \geqq 1}\left(\frac{|p|}{|q|+p^{2}}\right)^{\frac{2 m}{2-m}} d p d q$. On the other hand, we have

$$
\int_{p^{2}+q^{2}<1}|\hat{u}|^{m} d p d q \leqq C_{2}\left(\int_{p^{2}+q^{2}<1}|\hat{u}|^{2} d p d q\right)^{\frac{m}{2}} \leqq C_{2}\left(\int|\hat{u}|^{2} d p d q\right)^{\frac{m}{2}} .
$$

Again, $\int$ denotes the integral over $\mathbf{R}^{2}$. It follows then,

$$
\begin{aligned}
\int|\hat{u}|^{m} d p d q & \leqq C_{1}\left(\int\left|\widehat{u_{x}}\right|^{2}+\left|\widehat{D_{x}^{-1} u_{y}}\right|^{2} d p d q\right)^{\frac{m}{2}}+C_{2}\left(\int|\hat{u}|^{2} d p d q\right)^{\frac{m}{2}} \\
& \leqq C_{0}\left(\int\left|\widehat{u_{x}}\right|^{2}+\left|\widehat{D_{x}^{-1} u_{y}}\right|^{2}+|\hat{u}|^{2} d p d q\right)^{\frac{m}{2}} .
\end{aligned}
$$

By the theorem of Hausdorff-Young (p. 72 in [7]), we have

$$
\left(\int|u|^{n} d x d y\right)^{\frac{1}{n}} \leqq\left(\int|\hat{u}|^{m}\right)^{\frac{1}{m}},
$$


where $\frac{1}{n}+\frac{1}{m}=1$. Therefore, if $\frac{6}{5}<m<2$, we have $2<n<6$, and

$$
\left(\int_{\mathbf{R}^{2}}|u|^{n} d x d y\right)^{\frac{1}{n}} \leqq C_{0}\left(\int_{\mathbf{R}^{2}} u_{x}^{2}+\left(D_{x}^{-1} u_{y}\right)^{2}+u^{2} d x d y\right)^{\frac{1}{2}}
$$

Proof of Lemma 2. Let us assume that the box is of length $2 \pi \times 2 \pi$. The general case can be obtained by a scaling.

Consider the Fourier series representations of $u, u_{x}$ and $D_{x}^{-1} u_{y}$

$$
\begin{gathered}
u=\sum a_{m, n} e^{i m x+i n y}, \quad u_{x}=\sum b_{m, n} e^{i m x+i n y}, \\
D_{x}^{-1} u_{y}=\sum c_{m, n} e^{i m x+i n y} .
\end{gathered}
$$

Then,

$$
\int|u|^{2} d x d y=\sum_{m, n} a_{m, n}^{2}, \quad \int|\tilde{\nabla} u|^{2} d x d y=\sum_{m, n} b_{m, n}^{2}+c_{m, n}^{2}
$$

For some $0 \leqq \alpha \leqq 1$ and $p, q \neq 0$, we have

$$
\begin{aligned}
a_{m, n} & =\int e^{-i m x-i n y} u(x, y) d x d y \\
& =\alpha \int e^{-i m x-i n y} u(x, y) d x d y+(1-\alpha) \int e^{-i m x-i n y} u(x, y) d x d y \\
& =\frac{\alpha}{-i m} \int e^{-i p x-i q y} u_{x}(x, y) d x d y+\frac{(1-\alpha) m}{n} \int e^{-i m x-i n y} D_{x}^{-1} u_{y} d x d y .
\end{aligned}
$$

It follows that

$$
\begin{aligned}
\left|a_{m, n}\right| & \leqq \frac{\alpha}{|m|}\left|b_{m, n}\right|+\frac{(1-\alpha)|m|}{|n|}\left|c_{m, n}\right| \\
& \leqq \sqrt{\left(\frac{\alpha}{m}\right)^{2}+\left(\frac{(1-\alpha) m}{n}\right)^{2}} \cdot \sqrt{\left|b_{m, n}\right|^{2}+\left|c_{m, n}\right|^{2}}
\end{aligned}
$$

Let $\alpha=\frac{m^{2}}{|n|+m^{2}}$. Then

$$
\begin{aligned}
\left|a_{m, n}\right| & \leqq \frac{|m|}{|n|+m^{2}} \sqrt{\left|b_{m, n}\right|^{2}+\left|c_{m, n}\right|^{2}}, \\
\left|a_{m, n}\right|^{2} & \leqq\left(\frac{|m|}{|n|+m^{2}}\right)^{2}\left(\left|b_{m, n}\right|^{2}+\left|c_{m, n}\right|^{2}\right) .
\end{aligned}
$$

$\forall \varepsilon>0, \exists N_{\varepsilon}, M_{\varepsilon}$, s.t. for $n>N_{\varepsilon}, m>M_{\varepsilon}$, we have $\left(\frac{m}{n+m^{2}}\right)^{2}<\varepsilon$. Hence

$$
\begin{aligned}
\int u^{2} & =\sum_{m, n}^{\infty} a_{m, n}^{2} \leqq \sum_{m, n}^{N_{\varepsilon}, M_{\varepsilon}} a_{m, n}+\varepsilon\left(\sum_{m, n} b_{m, n}^{2}+c_{m, n}^{2}\right) \\
& =\sum_{m=1}^{M_{\varepsilon}} \sum_{n=1}^{N_{\varepsilon}}\left(\int_{\Omega} u w_{m, n}\right)+\varepsilon\left(\int_{\Omega} u_{x}^{2}+\left(D_{x}^{-1} u_{y}\right)^{2}\right) .
\end{aligned}
$$


Proof of Lemma 3. Since $\left\{u_{j}\right\}$ is a minimizing sequence, we have

$$
\begin{aligned}
1=\int\left|u_{j}\right|^{m+2} & =\int_{\left[\left|u_{j}\right| \geqq \frac{1}{\varepsilon}\right]}\left|u_{j}\right|^{m+2}+\int_{\left[\left|u_{j}\right| \leqq \varepsilon\right]}\left|u_{j}\right|^{m+2}+\int_{\left[\varepsilon<\left|u_{j}\right|<\frac{1}{\varepsilon}\right]}\left|u_{j}\right|^{m+2} \\
& \leqq \int_{\left[\left|u_{j}\right| \geqq \frac{1}{\varepsilon}\right]} \frac{\left|u_{j}\right|^{m+2+\gamma}}{\left|u_{j}\right|^{\gamma}}+\varepsilon^{m} \int_{\left[\left|u_{j}\right| \leqq \varepsilon\right]}\left|u_{j}\right|^{2}+\left(\frac{1}{\varepsilon}\right)^{m+2} \mu\left(\left[\left|u_{j}\right|>\varepsilon\right]\right) \\
& \leqq \varepsilon^{\gamma} \int_{\left[\left|u_{j}\right| \geqq \frac{1}{\varepsilon}\right]}\left|u_{j}\right|^{m+2+\gamma}+\varepsilon^{m} \int_{\left[\left|u_{j}\right| \leqq \varepsilon\right]}\left|u_{j}\right|^{2}+C_{\varepsilon} \mu\left(\left[\left|u_{j}\right|>\varepsilon\right]\right),
\end{aligned}
$$

where $0<\gamma<4-m$. From Lemma 1 , we have

$$
\int_{\left[\left|u_{j}\right| \geqq \frac{1}{\varepsilon}\right]}\left|u_{j}\right|^{m+2+\gamma} \leqq \int_{\mathbf{R}^{2}}\left|u_{j}\right|^{m+2+\gamma} \leqq C_{1},
$$

and

$$
\int_{\left[\left|u_{j}\right| \geqq \varepsilon\right]}\left|u_{j}\right|^{2} \leqq \int_{\mathbf{R}^{2}}\left|u_{j}\right|^{2} \leqq C_{2} .
$$

By choosing $\varepsilon$ small enough, we have

$$
\mu\left(\left[\left|u_{j}\right|>\varepsilon\right]\right) \geqq \frac{1-\varepsilon^{\gamma} C_{1}-\varepsilon^{m} C_{2}}{C_{\varepsilon}}=\delta .
$$

Proof of Lemma 4. For simplicity, we assume $|u|_{V} \leqq 1$. Consider any function $v$, such that $|v|_{V} \leqq 1$ and $|v|_{2} \neq 0$. Let $k=1+\frac{1}{|v|_{2}^{2}}$. We first prove that there is some $x$, such that

$$
\int_{B_{x}}\left(v^{2}+|\tilde{\nabla} v|^{2}\right) d x d y<m k \int_{B_{x}} v^{2} d x d y,
$$

where $B_{x}$ is the unit box centered at $x$ and $m$ is a certain integer.

If (17) is not true, then we can cover $\mathbf{R}^{2}$ with unit boxes $\left\{B_{x_{i}}\right\}$ so that each point $x$ is covered by at most $m$ unit boxes and we have

$$
\begin{aligned}
m \int_{\mathbf{R}^{2}}\left(v^{2}+|\tilde{\nabla} v|^{2}\right) d x d y & \geqq \sum_{i} \int_{B_{x_{i}}}\left(v^{2}+|\tilde{\nabla} v|^{2}\right) d x d y \geqq m k \sum_{i} \int_{B_{x_{i}}} v^{2} d x d y \\
& \geqq m k \int_{\mathbf{R}^{2}} v^{2} d x d y=m\left(1+\int_{\mathbf{R}^{2}} v^{2}\right)>m .
\end{aligned}
$$

Therefore

$$
|v|_{V}^{2}=\int_{\mathbf{R}^{2}}\left(v^{2}+|\tilde{\nabla} v|^{2}\right) d x d y>1,
$$

which is a contradiction. By Lemma 1 , we have, for $x$ satisfies (17) and some $p>2$,

$$
\begin{aligned}
\left(\int_{B_{x}}|v|^{p} d x d y\right)^{\frac{2}{p}} & \leqq C_{1} \int_{B_{x}}\left(|v|^{2}+|\tilde{\nabla} v|^{2}\right) d x d y<C_{1} m k \int_{B_{x}}|v|^{2} d x d y \\
& \leqq C_{1} m k\left(\int_{B_{x}}|v|^{p} d x d y\right)^{\frac{2}{p}}\left(\mu\left(B_{x} \cap \operatorname{supp}(v)\right)\right)^{\frac{p-2}{p}}
\end{aligned}
$$


and

$$
\mu\left(B_{x} \cap \operatorname{supp}(v)\right)>\left(\frac{1}{C_{1} m k}\right)^{\frac{p-2}{p}}
$$

Equation (19) is true for any $v$ satisfying $|v|_{v} \leqq 1$ and $|v|_{2} \neq 0$. Now let's take $v=\max \left(|u|-\frac{\varepsilon}{2}, 0\right)$, we have $|v|_{V} \leqq 1$ and $\int_{\mathbf{R}^{2}} v^{2} d x d y \geqq\left(\frac{\varepsilon}{2}\right)^{2} \delta$ and we have $k \leqq$ $1+\frac{1}{\left(\frac{\varepsilon}{2}\right)^{2} \delta}$. From (19), there exists $x$, s.t.

$$
\mu\left(B_{x} \cap \operatorname{supp}(v)\right)>\alpha, \quad \alpha=\left(\frac{1}{C_{1} m k}\right)^{\frac{p-2}{p}},
$$

i.e.

$$
\mu\left(B_{x} \cap[|u|>\varepsilon / 2]\right)>\alpha .
$$

The conclusion of the lemma follows with a shift.

\section{Stability of the Solitary Wave}

In this section, we show that the solitary wave of GKP-I is nonlinearly stable if $0<m<\frac{4}{3}$. In order to study the stability of solitary wave of GKP-I, we need to consider the local existence for GKP-I. There are many results on local existence for GKP-I (see [19, 20, 13]). For our purpose, we state here the local existence results by Saut [20]. Let

$$
\dot{H}_{x}^{-2}\left(\mathbf{R}^{2}\right)=\left\{f \in S^{\prime}\left(\mathbf{R}^{2}\right), \frac{1}{\xi_{1}^{2}} \hat{f}\left(\xi_{1}, \xi_{2}\right) \in L^{2}\left(\mathbf{R}^{2}\right)\right\}
$$

equipped with the norm

$$
\|f\|_{-2, x}=\left|\frac{1}{\xi_{1}^{2}} \hat{f}\right|_{2}
$$

and

$$
X_{s}=\left\{f \in H^{s}\left(\mathbf{R}^{2}\right), \mathscr{F}^{-1}\left(\frac{\hat{f}}{\xi_{1}}\right) \in H^{s}\left(\mathbf{R}^{2}\right)\right\},
$$

with

$$
\|f\|_{X_{s}}=\|f\|_{s}+\left\|\mathscr{F}^{-1}\left(\frac{\hat{f}}{\xi_{1}}\right)\right\|_{s}
$$

Theorem 2. Let $\phi \in X_{s}, s \geqq 3$, such that $\phi_{y y} \in \dot{H}_{x}^{-2}$. There exists $T>0$ such that (1) has a unique solution $u$ with $u(0)=\phi$ satisfying

$$
\begin{gathered}
u \in C\left([-T, T] ; H^{s}\left(\mathbf{R}^{2}\right)\right) \cup C^{1}\left([-T, T] ; H^{s-3}\left(\mathbf{R}^{2}\right)\right), \\
D_{x}^{-1} u_{y} \in C\left([-T, T] ; H^{s-1}\left(\mathbf{R}^{2}\right)\right) .
\end{gathered}
$$

Moreover, $Q(u)$ and $E(u)$ are well defined and independent of $t$.

We next give our definition of stability of the solitary waves. 
Definition 1. $A$ set $S \subset X$ is $X$-stable with respect to GKP-I if $\forall \varepsilon>0, \exists \delta>0$ such that for any $u_{0} \in X \cap X_{s}$ and $\partial_{y}^{2} u_{0} \in \dot{H}_{x}^{-2}, s \geqq 3$ with

$$
\inf _{v \in S}\left\|u_{0}-v\right\|_{X}<\delta,
$$

the solution $u(t)$ of (1) with $u(0)=v$ can be extended to a global solution in $C\left([0, \infty) ; X \cap X_{s}\right), s \geqq 3$ and

$$
\sup _{0 \leqq t<\infty} \inf _{v \in S}\|u(t)-v\|_{X}<\varepsilon .
$$

Otherwise $S$ is called $X$-unstable.

Now define the set of all ground state with speed $\omega>0$ as

$$
S_{\omega}=\left\{\varphi \in V\left(\mathbf{R}^{2}\right) ; K(\varphi)=I_{\omega}(\varphi)=(M(\omega))^{\frac{m+2}{m}}\right\} .
$$

Let $\varphi_{\omega}$ be a ground state of GKP-I. For simplicity, we denote $\varphi_{\omega}$ by $\varphi$. Then

$$
\omega \varphi+D_{x}^{-2} \varphi_{y y}-\varphi_{x x}-\varphi^{m+1}=0 .
$$

It is well known in [8] that the stability of the solitary waves depends on the behavior of the following functional:

$$
d(\omega)=E\left(\varphi_{\omega}\right)+\omega Q\left(\varphi_{\omega}\right) \quad \varphi_{\omega} \in S_{\omega} .
$$

It follows that

$$
\begin{aligned}
d(\omega) & =\frac{1}{2} I_{\omega}\left(\varphi_{\omega}\right)-\frac{1}{m+2} K\left(\varphi_{\omega}\right) \\
& =\frac{m}{2(m+2)} I_{\omega}\left(\varphi_{\omega}\right)=\frac{m}{2(m+2)} K\left(\varphi_{\omega}\right) .
\end{aligned}
$$

Theorem 3 (Nonlinear stability). Let $0<m<\frac{4}{3}$ with $m=m_{1} / m_{2}$, where $m_{1}$ is any even integer and $m_{2}$ any odd integer and $w>0$. Then $S_{\omega}$ is $V$-stable, where $V$ is defined in (3).

Remark 2. It is easy to calculate that

$$
d^{\prime \prime}(\omega)=\left(\frac{4-m}{2 m}\right)\left(\frac{4-3 m}{2 m}\right) \omega^{\frac{4-m}{2 m}-2} A,
$$

where $A=\frac{1}{3} \iint\left(\varphi_{x}^{2}+\left(D_{x}^{-1} \varphi_{y}\right)^{2}\right) d x d y$. Hence

$$
d^{\prime \prime}(\omega)>0 \Leftrightarrow 0<m<\frac{4}{3} .
$$

In order to prove Theorem 3, we need several lemmas.

Lemma 5. $d(\omega)$ is differentiable and strictly increasing for $\omega>0,0<m<4$ with $m=m_{1} / m_{2}$, where $m_{1}$ is any even integer and $m_{2}$ any odd integer. 
Proof. In fact, from (5), we have

$$
d(\omega)=\frac{m}{2(m+2)} \omega^{\frac{4-m}{2 m}}(M(1))^{\frac{m+2}{m}}
$$

and

$$
d^{\prime}(w)=\frac{4-m}{4(m+2)} w^{\frac{4-3 m}{2 m}}(M(1))^{\frac{m+2}{m}}>0
$$

for $0<m<4$.

Lemma 6. Let $d^{\prime \prime}(\omega)>0$ with $\omega>0$. Then $\exists \varepsilon>0$, such that for $\omega_{1}>0$ with $\left|\omega_{1}-\omega\right|<\varepsilon$ we have

$$
d\left(\omega_{1}\right) \geqq d(\omega)+d^{\prime}(\omega)\left(\omega_{1}-\omega\right)+\frac{1}{4} d^{\prime \prime}(\omega)\left|\omega-\omega_{1}\right|^{2} .
$$

Proof. This follows by Taylor's expansion at $\omega_{1}=\omega$.

Define

$$
U_{\omega, \varepsilon}=\left\{u \in V\left(\mathbf{R}^{2}\right) ; \inf _{\varphi \in S_{\omega}}\|u-\varphi\|_{V}<\varepsilon\right\} .
$$

Since $d(\omega)$ is differentiable and strictly increasing for $\omega>0$, it follows that for $u$ near $\varphi$ and $\varphi \in S_{\omega}$,

$$
\omega(u)=d^{-1}\left(\frac{m}{2(m+2)} K(u)\right)
$$

is a $C^{1}$ map:

$$
\omega(\cdot): U_{\omega, \varepsilon} \rightarrow \mathbf{R}^{+} \text {for small } \varepsilon>0,
$$

and $\omega\left(\varphi_{\omega}\right)=\omega$ for any $\varphi_{\omega} \in S_{\omega}$.

The next lemma uses the variational characterization of ground states to establish the key inequality in the proof of stability.

Lemma 7. Suppose $d^{\prime \prime}(\omega)>0$ for $\omega>0$. Then there exists $\varepsilon>0$ such that for all $u \in U_{\omega, \varepsilon}$ and $\varphi_{\omega} \in S_{\omega}$,

$$
E(u)-E\left(\varphi_{\omega}\right)+\omega(u)\left(Q(u)-Q\left(\varphi_{\omega}\right)\right) \geqq \frac{1}{4} d^{\prime \prime}(\omega)|\omega(u)-\omega|^{2},
$$

where $\omega(u)$ is defined by

$$
\omega(u)=d^{-1}\left(\frac{m}{2(m+2)} K(u)\right) \quad \text { for } u \in U_{\omega, \varepsilon} .
$$

Proof. First of all, we have

$$
E(u)+\omega(u) Q(u)=\frac{1}{2} I_{\omega(u)}(u)-\frac{1}{m+2} K(u) .
$$

Since

$$
\frac{2(m+2)}{m} d(\omega(u))=K(u),
$$

and

$$
\frac{2(m+2)}{m} d(\omega(u))=K\left(\varphi_{\omega(u)}\right), \quad \varphi_{\omega(u)} \in S_{\omega(u)},
$$


then

$$
K(u)=K\left(\varphi_{\omega(u)}\right)
$$

This implies that

$$
I_{\omega(u)}(u) \geqq I_{\omega(u)}\left(\varphi_{\omega(u)}\right) .
$$

Since $\varphi_{\omega(u)}$ is a minimizer of $I_{\omega(u)}$ subject to the constraint $K(u)=K\left(\varphi_{\omega(u)}\right)$ and $\omega(u) \in C^{1}$, then by (27) and Lemma 6 we have

$$
\begin{aligned}
E(u)+\omega(u) Q(u) & \geqq \frac{1}{2} I_{\omega(u)}\left(\varphi_{\omega(u)}\right)-\frac{1}{m+2} K\left(\varphi_{\omega(u)}\right)=d(\omega(u)) \\
& \geqq d(\omega)+d^{\prime}(\omega)(\omega(u)-\omega)+\frac{1}{4} d^{\prime \prime}(\omega)|\omega(u)-\omega|^{2} \\
& =E\left(\varphi_{\omega}\right)+\omega(u) Q\left(\varphi_{\omega}\right)+\frac{1}{4} d^{\prime \prime}(\omega)|\omega(u)-\omega|^{2},
\end{aligned}
$$

where we use the fact

$$
d^{\prime}(\omega)=Q\left(\varphi_{\omega}\right)
$$

Now we can prove Theorem 3.

Proof. Assume that $S_{\omega}$ is V-unstable. Then by the definition of stability, $\exists \delta>0$ and initial data $u_{k}(0) \in U_{\omega, \frac{1}{k}}$ such that

$$
\sup _{t>0} \inf _{\varphi \in S_{\omega}}\left\|u_{k}(t)-\varphi\right\|_{V} \geqq \delta,
$$

where $u_{k}(t)$ is the solution of GKP-I with initial data $u_{k}(0)$. By continuity in $t$, we can pick the first time $t_{k}$ so that

$$
\inf _{\varphi \in S_{\omega}}\left\|u_{k}\left(t_{k}\right)-\varphi\right\|_{V}=\delta
$$

Since $E(u)$ and $Q(u)$ are conserved at $t$ and continuous for $u$, we can find $\varphi_{k} \in S_{\omega}$ such that

$$
\left|E\left(u_{k}\left(t_{k}\right)\right)-E\left(\varphi_{k}\right)\right|=\left|E\left(u_{k}(0)\right)-E\left(\varphi_{k}\right)\right| \rightarrow 0
$$

as $k \rightarrow \infty$ and

$$
\left|Q\left(u_{k}\left(t_{k}\right)\right)-Q\left(\varphi_{k}\right)\right|=\left|Q\left(u_{k}(0)\right)-Q\left(\varphi_{k}\right)\right| \rightarrow 0
$$

as $k \rightarrow \infty$. Choose $\delta$ small enough so that Lemma 7 applies,

$$
E\left(u_{k}\left(t_{k}\right)\right)-E\left(\varphi_{k}\right)+\omega\left(u_{k}\left(t_{k}\right)\right)\left(Q\left(u_{k}\left(t_{k}\right)\right)-Q\left(\varphi_{k}\right)\right) \geqq \frac{1}{4} d^{\prime \prime}(\omega)\left|\omega\left(u_{k}\left(t_{k}\right)\right)-\omega\right|^{2} .
$$

By (32), there exists $\psi_{k} \in S_{\omega}$ such that

$$
\begin{aligned}
\left\|u_{k}\left(t_{k}\right)\right\|_{V} & \leqq\left\|\varphi_{k}\right\|_{V}+2 \delta \\
& \leqq\left(1+\frac{1}{\omega}\right) I_{\omega}\left(\varphi_{k}\right)+2 \delta \\
& \leqq c(\omega) M(\omega)^{\frac{m+2}{m}}+2 \delta<+\infty
\end{aligned}
$$


Since $\omega(u)$ is a continuous map, $\omega\left(u_{k}\left(t_{k}\right)\right)$ is uniformly bounded for $k$. By (35), letting $k \rightarrow \infty$, we have

$$
\omega\left(u_{k}\left(t_{k}\right)\right) \rightarrow \omega
$$

Hence

$$
\lim _{k \rightarrow \infty} K\left(u_{k}\left(t_{k}\right)\right)=\lim _{k \rightarrow \infty} \frac{2(m+2)}{m} d\left(\omega\left(u_{k}\left(t_{k}\right)\right)=\frac{2(m+2)}{m} d(\omega) .\right.
$$

On the other hand,

$$
\begin{aligned}
I_{\omega}\left(u_{k}\left(t_{k}\right)\right) & =2\left(E\left(u_{k}\left(t_{k}\right)\right)+\omega Q\left(u_{k}\left(t_{k}\right)\right)+\frac{2}{m+2} K\left(u_{k}\left(t_{k}\right)\right)\right. \\
& =2 d\left(\omega\left(u_{k}\left(t_{k}\right)\right)+2\left(\omega-\omega\left(u_{k}\left(t_{k}\right)\right) Q\left(u_{k}\left(t_{k}\right)\right)+\frac{2}{m+2} K\left(u_{k}\left(t_{k}\right)\right) .\right.\right.
\end{aligned}
$$

Since

$$
Q\left(u_{k}\left(t_{k}\right)\right)=Q\left(u_{k}(0)\right) \leqq\left\|u_{k}\left(t_{k}\right)\right\|_{V} \leqq C(\omega)<+\infty,
$$

then by (39)

$$
I_{\omega}\left(u_{k}\left(t_{k}\right)\right) \rightarrow 2 d(\omega)+\frac{2}{m+2} \frac{2(m+2)}{m} d(\omega)=\frac{2(m+2)}{m} d(\omega) \quad \text { as } k \rightarrow \infty .
$$

That is

$$
I_{\omega}\left(u_{k}\left(t_{k}\right)\right) \rightarrow I\left(\varphi_{\omega}\right)=(M(\omega))^{\frac{m+2}{m}}
$$

Let

$$
v_{k}\left(t_{k}\right)=\left(K\left(u_{k}\left(t_{k}\right)\right)\right)^{-\frac{1}{m+2}} u_{k}\left(t_{k}\right) .
$$

Then $K\left(v_{k}\left(t_{k}\right)\right)=1$ and

$$
\begin{aligned}
I_{\omega}\left(v_{k}\left(t_{k}\right)\right) & =\left(K\left(u_{k}\left(t_{k}\right)\right)\right)^{-\frac{2}{m+2}} I_{\omega}\left(u_{k}\left(t_{k}\right)\right) \\
& \rightarrow \frac{(M(\omega))^{\frac{m+2}{m}}}{\left(\frac{2(m+2)}{m} d(\omega)\right)^{\frac{2}{m+2}}}=(M(\omega))^{\frac{m+2}{m}}(M(\omega))^{-\frac{2}{m}}=M(\omega) .
\end{aligned}
$$

Hence, $v_{k}\left(t_{k}\right)$ is a minimizing sequence. Therefore, $\exists \varphi_{k} \in S_{\omega}$ such that

$$
\lim _{k \rightarrow \infty}\left\|v_{k}\left(t_{k}\right)-(M(\omega))^{-\frac{1}{m}} \varphi_{k}\right\|_{V}=0
$$

where $K\left((M(\omega))^{-\frac{1}{m}} \varphi_{k}\right)=1$. This implies that

$$
\begin{aligned}
& \lim _{k \rightarrow \infty}\left\|u_{k}\left(t_{k}\right)-\varphi_{k}\right\|_{V}=\lim _{k \rightarrow \infty}\left[\left(K\left(u_{k}\left(t_{k}\right)\right)\right)^{\frac{1}{m+2}} \cdot\left\|\left(K\left(u_{k}\left(t_{k}\right)\right)\right)^{-\frac{1}{m+2}}\left(u_{k}\left(t_{k}\right)-\varphi_{k}\right)\right\|_{V}\right] \\
& \leqq M^{\frac{1}{m}}(\omega)\left[\lim _{k \rightarrow \infty}\left\|v_{k}\left(t_{k}\right)-M^{-\frac{1}{m}}(\omega) \varphi_{k}\right\|_{V}\right] \\
& \quad+\lim _{k \rightarrow \infty}\left|M^{\frac{-1}{m}}(\omega)-\left(K\left(u_{k}\left(t_{k}\right)\right)\right)^{-\frac{1}{m+2}}\right|\left\|\varphi_{k}\right\|_{V}=0
\end{aligned}
$$

since $\left\|\varphi_{k}\right\|_{V}^{2} \leqq\left(1+\frac{1}{\omega}\right) I_{\omega}\left(\varphi_{k}\right) \rightarrow\left(1+\frac{1}{\omega}\right)(M(\omega))^{\frac{m+2}{m}}<+\infty$.

Hence (45) contradicts with (32). 


\section{References}

1. Ablowitz, M., Segur, H.: Soliton and the Inverse Scattering Transform. Philadelphia: SIAM Stud. Appl. Math. 1981

2. Ablowitz, M., Clarkson, P.: Solitons, Nonlinear Evolution Equations, and Inverse Scattering. Cambridge: Cambridge U.P., 1991

3. Shatah, J., Strauss, W.: Instability of nonlinear bound states. Commun. Math. Phys. 100, 173 (1985)

4. Shatah, J.: Stable Klein-Gordon Equations. Commun. Math. Phys. 91, 313-327 (1983)

5. Levandosky, S.: Stability and instability of fourth order solitary waves. To appear

6. Kadomtsev, B.B., Petviashvili, V.I.: On the stability of solitary waves in weakly dispersing media. Sov. Phy. Dokl. 15, 539 (1970)

7. Champeney, D.C.: A handbook of Fourier Theorems. Cambridge: Cambridge University Press, 1987

8. Grillakis, M., Shatah, J., Strauss, W.: Stability theory of solitary waves in the presence of symmetry. J. Funct. Anal. 74, 160-197 (1987)

9. Turitsyn, S., Fal'kovich, G.: Stability of magnetoelastic soliton and self-focusing of sound in antiferromagnet. Sov. Phys. JETP 62 (1) July, 1985

10. Ablowitz, M., Villarroel, J.: On the Kadomtsev-Petviashvili equation and associated constraints. Stud. Appl. Math. 85, 195-213 (1991)

11. Schwarz, M.: Periodic solutions of Kadomtsev-Petviashvili equation. Adv. in Math. 66, 217-233 (1987)

12. Boiti, M., Pempinelli, F., Pogrebkov, A.: Solutions of the KPI equation with smooth initial data. Inverse Problems 10, no. 3, 505-519 (1994)

13. Bourgain, J.: On the Cauchy problem for the Kadomtsev-Petviashvili equations. Geom. Funct. Anal. 3(4), 315-341

14. Wang, X.P., Ablowitz, M., Segur, H.: Wave collapse and instability of solitary waves of a generalized nonlinear Kadomtsev-Petviashvili equation. Physica D 78, 241-265 (1994)

15. Brezis, H., Lieb, E.: Minimum action solutions of some vector field equation. Commun. Math. Phys. 96, 97-113 (1984)

16. Brezis, H., Lieb, E.: A relation between pointwise convergence of functions and convergence of functionals. Proc. Amer. Math. Soc. 88, 437-477 (1983)

17. Struwe, M.: Variational Methods. N.Y., N.Y: Springer-Verlag, 1990

18. Lieb, E.: On the lowest eigenvalue of the Laplacian for the intesection of two domains. Invent. Math. 74, 441-448 (1983)

19. Ukai, S.: Local solutions of the Kadomtsev-Petviashvili equation. J. Fac. Sci. Univ. Tokyo Sect. IA Math. 36, 193-209 (1989)

20. Saut, J.C.: Remarks on the generalized Kadomtsev-Petviashvili equations. Indiana Univ. Math. J. 42, No. 3, 1011-1026 (1993)

21. de Bouard, A., Saut, J.C.: Solitary waves of generalized Kadomtsev-Petviashvili equation. Preprint 1995

22. Colliander, J.: Globalizing estimates for the periodic KPI equation. Preprint 1995

23. Lions, P.L.: The concentration-compactness principle in the calculus of variations, The locally compact case, I and II. Ann. I.H.P. Analyse Non-Lineaire, I, 104-145, 223-283 (1984)

24. de Bouard, A., Saut, J.C.: Remarks on the stability of generalized KP solitary waves. Preprint 1996

Communicated by A. Jaffe 